\title{
Comparison of Neutrophil Gelatinase-associated Lipocalin and Renal Resistive Index as Acute Kidney Injury Predictor in Critically III Patients at ICU H. Adam Malik Hospital Medan
}

\author{
Muhammad Aldi Rivai Ginting*, Achsanuddin Hanafie, Bastian Lubis \\ Department of Anesthesia and Intensive Care Medical, Sumatera Utara University, H. Adam Malik General Hospital Medan, \\ North Sumatra, Indonesia
}

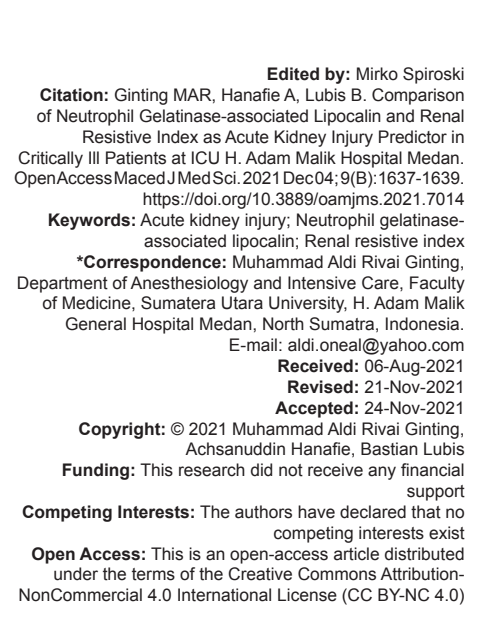

Introduction

Acute kidney injury (AKI) is a condition that describes as a broad spectrum from mild kidney function impairment to permanent kidney damage and complete loss of kidney function. AKI is a complication that is often found in critically ill patients treated in the Intensive Care Unit (ICU) [2], Neutrophil gelatinaseassociated lipocalin (NGAL) is a glycoprotein that is stored in mature neutrophil granules and released by tubular kidney cells after an acute tubular damage.

Laboratory tests can detect an increase in NGAL several hours after tubular damage and are reported to predict AKI earlier than serum creatinine [1], [4], [8]. Apart from NGAL, Renal resistive index (RRI) also been reported to be a good early marker of AKI. In contrast to NGAL which is examined from blood, RRI is a sonographic index. Renal vasoconstriction is an early manifestation of AKI. Doppler ultrasound can measure $\mathrm{RRI}$ show changes in intrarenal or interlobar arcuate arteries blood flow profile [6].

\section{Methods}

This study is an observational analytic study with a prospective cohort design. Conducted at ICU of Haji Adam Malik Hospital Medan in April until May 2021 after obtaining approval from Health Research Ethics Commission and approved by the Ethics Committee of Medical Faculty, Sumatera Utara University and H. Adam Malik Hospital Medan. Sample in this study is 40 subjects. The sampling technique used non-probability sampling with consecutive sampling, where all subjects who came and met inclusion and exclusion criteria. Inclusion criteria in this study are patients aged 18-65 years and patients who met criteria for critically ill patients according to the Ministry of Health regulations with $1^{\text {st }}$ and $2^{\text {nd }}$ priority level, while exclusion criteria are patients with acute and chronic renal failure, patients receiving nephrotoxic drug therapy, and patients with malignancy. The study sample collection was in accordance with inclusion and exclusion criteria in critically ill and septic patients, who were admitted to the ICU at H. Adam Malik Hospital Medan. 
Basic data collection such as gender, age, admission diagnosis, laboratory results, and urine output monitoring by volunteers. Renal Doppler ultrasound performed to assess RRI) (performed by researcher and confirmed by ICU supervisor) and urine NGAL collected within $3 \mathrm{~h}$ of ICU admission. Then, urine output and creatinine clearance were monitored to assess whether AKI occurred according to the RIFLE criteria. Re-examination of urea and creatinine levels was carried out within $24 \mathrm{~h}$ of treatment.

\section{Results}

Characteristics of subjects, from 40 samples, number of male samples are $26(66 \%)$ and female samples are $14(35 \%), p=0.001$. Mean NGAL value sample are $80.35 \pm 504.61 \mathrm{ng} / \mathrm{dL}$ with $\mathrm{p}=0.01$. Meanwhile, mean value of $\mathrm{RRI}$ are $0.68 \pm 0.088$ $(p=0.038)$, statistically not normally distributed. Mean value $B M I$ in this study are $25.05 \pm 2.214, p=0.402$. Mean age are $48.35 \pm 14.143$ years, $p=0.001$. And incidence of AKI in this study are 21 (52.5\%) samples.

There is a statistically and clinically difference in mean value of RRI between $A K I$ and non-AKI groups, $0.719 \pm 0.060$ compared to $0.060 \pm 0.077$, with $p=0.001$. Table 1: Predictive value of RRI

\begin{tabular}{llllllllll}
\hline$(\%)$ & TP & TN & FP & FN & Sensitivity & Specificity & Accuracy & PPV & NPV \\
\hline RRI & 6 & 3 & 16 & $15(71.4)$ & 71 & 84 & 87 & 78.9 & 27
\end{tabular}

$$
\begin{aligned}
& (28.6) \quad(15.8) \quad(84.2) \\
& \hline \text { TP: True positive, TN: True negative, FP: False positive, FN: False negative, PPV: Positive predictive value, } \\
& \text { NPV: Negative predictive value. RRI: Renal resistive index. }
\end{aligned}
$$

Table 1 describes predictive value of RRI on predicting $\mathrm{AKI}$ in ICU patients. In this study, value of True Positive was $28.6 \%$, True Negative $15.8 \%$, False Positive $84.2 \%$, and False Negative $71.4 \%$. RRI has a sensitivity of $71 \%$, specificity of $84 \%$, and accuracy of $87 \%$ in predicting AKI with AUROC = 0.873 (Figure 1). Meanwhile, Table 2 shows predictive value of NGAL on predicting AKI. Obtained a True Positive value of $66.7 \%$, True Negative $89.5 \%$, False Positiv e 10.5\%, and False Negative 33.3\%, with AUROC = 0.781 (Figure 2).

\section{Table 2: Predictive value of NGAL} \begin{tabular}{llllllllll}
\hline$(\%)$ & TP & TN & FP & FN & Sensitivity & Specificity & Accuracy & PPV & NPV \\
\hline NGAL & 14 & 17 & 2 & 7 & 66 & 89 & 78 & 87 & 29 \\
& $(66.7)$ & $(89.5)$ & $(10.5)$ & $(33.3)$ & & & & &
\end{tabular}

TP: True positive, TN: True negative, FP: False positive, FN: False negative, PPV: Positive predictive value, NPV: Negative predictive value. NGAL: Neutrophil gelatinase-associated lipocalin.

\section{Discussion}

In our study, it found that RRI had a sensitivity of $71 \%$ and a specificity of $84 \%$ with an accuracy value of $87 \%$. When compared with a study conducted by Muthukrishnan et al. (2019) where sensitivity is $57.1 \%$,

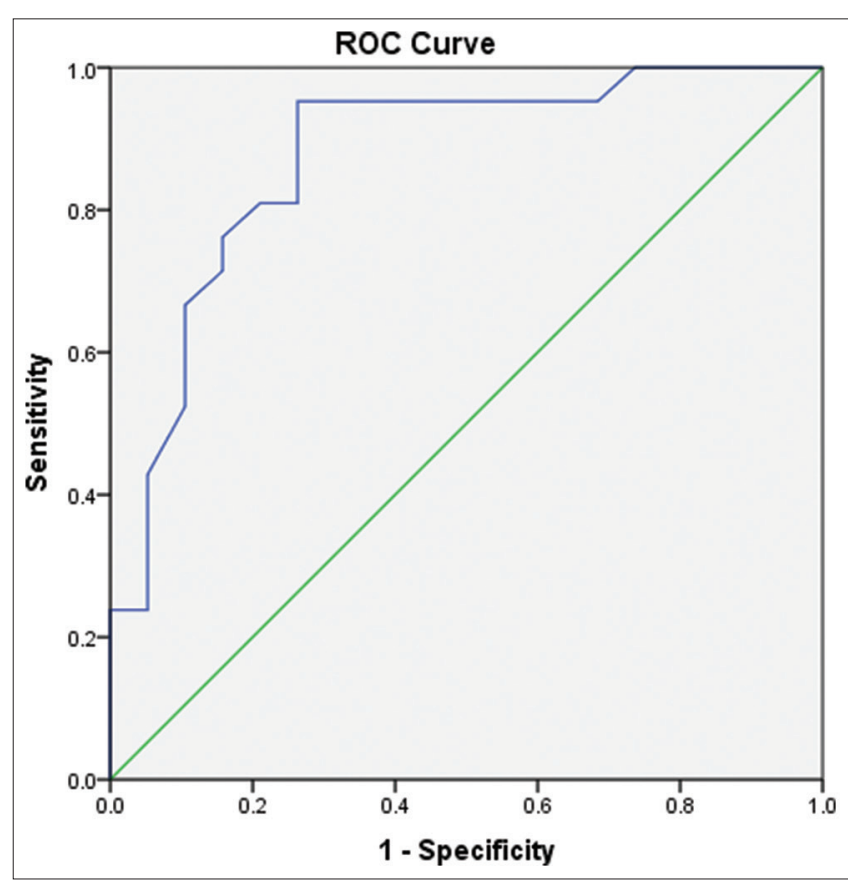

Figure 1: Renal resistive ROC curve

specificity $85.5 \%$, accuracy $77 \%$, and study conducted by Mulier et al. (2018) which sensitivity 53\%, and specificity are $87 \%$ [7]. RRI value in our study was not much different. In addition, this study also found significant differences, where the RRI value was higher in AKI subjects. Therefore, Renal Doppler examination is a reliable examination and an easier assessment in assessing renal perfusion in critically ill patients.

NGAL has a $66 \%$ sensitivity and $89 \%$ specificity, with $78 \%$ accuracy. This is in accordance with research

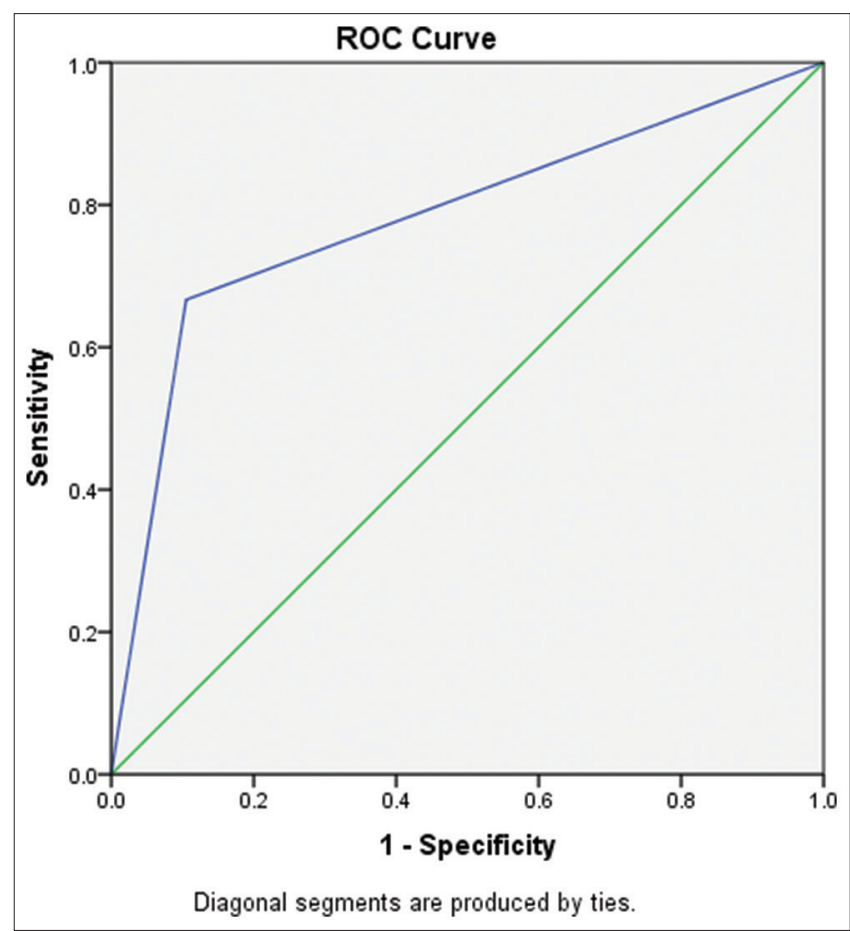

Figure 2: Neutrophil gelatinase-associated lipocalin ROC curve 
conducted by Khawaja et al. (2019) which obtained a sensitivity of $78.5 \%$, specificity of $88.8 \%$, and study of Mahadevaiah et al. (2021) which obtained a $83.6 \%$ sensitivity and a $88.9 \%$ specificity. So, NGAL value in this study is not that much different. In addition, in this study, NGAL value was statistically significant and could predict incidence of AKI [3].

Based on the results of our study, it is known that RRI has better sensitivity than NGAL in predicting incidence of AKI in critically ill patients. However, a high NGAL value can also be a marker of AKI, because it has a high sensitivity. This is because NGAL is a glycoprotein that stored in mature neutrophil granules and found to be released by renal tubular cells after acute tubular damage. Laboratory examination can detect an increase in NGAL several hours after the occurrence of tubular damage [1], [3], [5].

Limitations in our study is RRI and NGAL cannot measure mortality rate because in this study there is no observations on outcome of specified samples.

\section{Conclusion}

RRI has better sensitivity than NGAL in predicting incidence of AKI. However, RRI and NGAL can be a good marker to predict incidence of AKI.

\section{References}

1. Banai A, Rozenfeld K, Levit D, Merdler I, Loewenstein I, Banai S, et al. Neutrophil gelatinase-associated lipocalin
(NGAL) for the prediction of acute kidney injury in chronic kidney disease patients treated with primary percutaneous coronary intervention. IJC Heart Vasc. 2021;32:1-5. https://doi. org/10.1016/j.ijcha.2020.100695

PMid:33364336

2. Case J, Khan S, Khalid R, Khan A. Epidemiology of acute kidney injury in the intensive care unit. Crit Care Res Pract. 2013;2013:479730. https://doi.org/10.1155/2013/479730 PMid:23573420

3. Khawaja S, Jafri L, Siddiqui I, Hashmi M, Ghani F. The utility of neutrophil gelatinase-associated Lipocalin (NGAL) as a marker of acute kidney injury (AKI) in critically III patients. Biomarker Res. 2019;7:4. https://doi.org/10.1186/ s40364-019-0155-1

PMid:30834123

4. Li H, Yu Z, Gan L, Peng L, Zhou Q. Serum NGAL and FGF23 may have certain value in early diagnosis of CIN. Renal Failure. 2018;40(1):547-53. https://doi.org/10.1080/08860 22X.2018.1487860

PMid:30278796

5. Mahadevaiah M, Thimmaiah M, Yerramsetty V, Kumar J, Kumar R. Diagnostic relevance of neutrophil gelatinase associated lipocalin in early detection of acute kidney injury. J Emerg Pract Trauma. 2021;7(2):88-92.

6. Mulier J, Rozemeijer S, Röttgering JG, Spoelstra-de Man AM, Elbers PW, Tuinman PR, et al. Renal resistive index as an early predictor and discriminator of acute kidney injury in critically ill patients; a prospective observational cohort study. PLoS One. 2018;13(6):1-18. https://doi.org/10.1371/journal. pone.0197967

PMid:29889830

7. Muthukrishnan K, Parida S, Barathim S, Badhe A, Mishra S Doppler resistive index to reflect risk of acute kidney injury after major abdominal surgery: A prospective observational trial. Indian J Anaesth. 2019;63(7):551-7. https://doi.org/10.4103/ija. IJA_189_19

PMid:31391618

8. Shang $W$, Wang $Z$. The update of NGAL in acute kidney injury. Curr Protein Peptide Sci. 2017;17:1211-7. https://doi.org/10.217 4/1389203717666160909125004

PMid:27634444 